\title{
AN APPROACH TO PILLAI'S PROBLEM WITH THE PELL SEQUENCE AND THE POWERS OF 3
}

\author{
A. ÇAĞMAN
}

Received 18 January, 2021

\begin{abstract}
In this paper, we consider the Diophantine equation $P_{n}-3^{a}=v$ and find all $v$ having at least two representations. In the proof of the main theorem, we use a version of the BakerDavenport reduction method.
\end{abstract}

2010 Mathematics Subject Classification: 11B39; $11 \mathrm{~J} 86$

Keywords: Pillai's problem, Pell numbers, Diophantine equation, Baker's theory

\section{INTRODUCTION}

Let us consider the Diophantine equation

$$
a^{x}-b^{y}=v
$$

where $a, b$ are given fixed positive integers, $v \geq 1$ and $\min \{x, y\} \geq 2$. This equation is also known as Pillai's equation. The $v=1$ case, known also as the Catalan's conjecture, was proved by Mihăilescu [24]. In 1936 and 1937 (see [25, 26]), Pillai conjectured that only integers $v$ with at least two representations of the form $2^{x}-3^{y}$ are as follows:

$$
2^{3}-3^{2}=-1=2^{1}-3^{1}, \quad 2^{5}-3^{3}=5=2^{3}-3^{1}, \quad 2^{8}-3^{5}=13=2^{4}-3^{1} .
$$

This conjecture is a continuation of the work of Herschfeld [20,21] in 1935 and verified by Stroeker and Tijdeman in 1982 [28]. The problem that Pillai deals with in the general case has recently begun to be studied in integer sequences thanks to Baker's theory. For example in [13], authors shows that the set of integers admitting at least two representations of the form $F_{n}-2^{a}$ is $\{-30,-11,-3,-1,0,1,5,85\}$. Since then, many studies including integer sequences were handled by the authors (see [3, 6-12, 15-19]).

We consider Pell sequence which is defined by $P_{0}=0, P_{1}=1$ and $P_{n}=2 P_{n-1}+P_{n-2}$. Some of the terms of the Pell sequence are given by

$$
0,1,2,5,12,29,70, \ldots
$$


Its characteristic polynomial is of the form $x^{2}-2 x-1=0$ whose roots are $\alpha=1+\sqrt{2}$ and $\beta=1-\sqrt{2}$. Binet's formula enables us to rewrite the Pell sequence by using the roots $\alpha$ and $\beta$ as

Also, it is known that

$$
P_{n}=\frac{\alpha^{n}-\beta^{n}}{2 \sqrt{2}}
$$

$$
\alpha^{n-2} \leq P_{n} \leq \alpha^{n-1} .
$$

More detailed information on Pell sequences can be found in [22].

In this article, we aimed to prove the following result.

Theorem 1. The only integers $v$ having at least two representations of the form

$$
P_{n}-3^{a}=v
$$

are $\{-1,2\}$. Furthermore, all the representations of these integers as $P_{n}-3^{a}$ with integers $n \geq 0$ and $a \geq 0$ are given by

$$
\begin{aligned}
& P_{2}-3^{1}=-1=P_{0}-3^{0} \\
& P_{5}-3^{3}=2=P_{3}-3^{1} .
\end{aligned}
$$

\section{PRELIMINARIES}

\subsection{Linear forms in logarithms.}

Let us give the concept of logarithmic height of an algebraic number and its some properties, which is a component of an important theorem that we will use for proof.

Definition 1. Let $\xi$ be an algebraic number of degree $d$ with minimal polynomial

$$
a_{0} x^{d}+a_{1} x^{d-1}+\cdots+a_{d}=a_{0} \cdot \prod_{i=1}^{d}\left(x-\xi_{i}\right)
$$

where $a_{i}$ 's are relatively prime integers with $a_{0}>0$ and $\xi_{i}$ 's are conjugates of $\xi$. Then

$$
h(\xi)=\frac{1}{d}\left(\log a_{0}+\sum_{i=1}^{d} \log \left(\max \left\{\left|\xi_{i}\right|, 1\right\}\right)\right)
$$

is called the logarithmic height of $\xi$. The following proposition gives some properties of logarithmic height that can be found in [27].

Proposition 1. Let $\xi, \xi_{1}, \xi_{2}, \ldots, \xi_{t}$ be elements of an algebraic closure of $\mathbb{Q}$ and $m \in \mathbb{Z}$. Then

(1) $h\left(\xi_{1} \ldots \xi_{t}\right) \leq \sum_{i=1}^{t} h\left(\xi_{i}\right)$

(2) $h\left(\xi_{1}+\cdots+\xi_{t}\right) \leq \log t+\sum_{i=1}^{t} h\left(\xi_{i}\right)$

(3) $h\left(\xi^{m}\right)=|m| h(\xi)$. 
We use a Baker-type lower bound (see in [2]) for a nonzero linear form in logarithms of algebraic numbers several times to prove our main result Theorem 1. In the literature, there are many such bounds but we will use the result of Matveev stated in the following theorem (see [23] or Theorem 9.4 in [5]).

Theorem 2. Let $\gamma_{1}, \gamma_{2}, \ldots, \gamma_{s}$ be nonzero elements of a real algebraic number field F of degree $D, b_{1}, b_{2}, \ldots, b_{s}$ rational integers. Set

$$
\Lambda:=\gamma_{1}^{b_{1}} \ldots \gamma_{s}^{b_{s}}-1
$$

and

$$
B:=\max \left\{\left|b_{1}\right|, \ldots,\left|b_{s}\right|\right\}
$$

If $\Lambda$ is nonzero, then

$$
\log |\Lambda|>-3 \cdot 30^{s+4} \cdot(s+1)^{5.5} \cdot D^{2} \cdot(1+\log D) \cdot(1+\log (s B)) \cdot A_{1} \cdots A_{s}
$$

where

$$
A_{i} \geq \max \left\{D \cdot h\left(\gamma_{i}\right),\left|\log \gamma_{i}\right|, 0.16\right\}
$$

for all $1 \leq i \leq s$. If $\mathbb{F}=\mathbb{R}$, then

$$
\log |\Lambda|>-1.4 \cdot 30^{s+3} \cdot t^{4.5} \cdot D^{2} \cdot(1+\log D) \cdot(1+\log B) \cdot A_{1} \cdots A_{s} .
$$

\subsection{Baker-Davenport reduction lemma.}

We use some of the results of the continued fractions theory to reduce the upper bounds on variables, which are quite large, that we have obtained throughout the calculations. The following lemma we specifically use, and taken from [4], is a variation of the result due to [14] which is a generalization of a result of [1].

Lemma 1. Let $A, B, \mu$ be some real numbers with $A>0$ and $B>1$ and let $\gamma$ be an irrational number and $M$ be a positive integer. Take $p / q$ as a convergent of the continued fraction of $\gamma$ such that $q>6 M$. Set $\varepsilon:=\|\mu q\|-M\|\gamma q\|>0$ where $\|\cdot\|$ denotes the distance from the nearest integer. Then there is no solution to the inequality

$$
0<|u \gamma-v+\mu|<A B^{-w}
$$

in positive integers $u$, $v$ and $w$ with

$$
u \leq M \quad \text { and } \quad w \geq \frac{\log \frac{A q}{\varepsilon}}{\log B} .
$$

\section{Proof of Theorem 1}

Let us assume that there exist nonnegative integers $n, a, n_{1}, a_{1}$ such that $(n, a) \neq\left(n_{1}, a_{1}\right)$ and

$$
P_{n}-3^{a}=P_{n_{1}}-3^{a_{1}}
$$


If we take $a=a_{1}$ then we obtain $P_{n}=P_{n_{1}}$ and so $(n, a)=\left(n_{1}, a_{1}\right)$ which contadicts our assumption. Thus, without loss of the generality, we can assume that $a>a_{1}$. By rewriting the equation (3.1) as

$$
P_{n}-P_{n_{1}}=3^{a}-3^{a_{1}}
$$

we get $n>n_{1}$ from the positivity of left hand side of the equality. Using the equality above and the inequality (1.3), we obtain

$$
\begin{aligned}
& \alpha^{n-4} \leq P_{n-2} \leq P_{n}-P_{n_{1}}=3^{a}-3^{a_{1}}<3^{a}, \\
& \alpha^{n-1} \geq P_{n}>P_{n}-P_{n_{1}}=3^{a}-3^{a_{1}} \geq 3^{a-1} .
\end{aligned}
$$

These two inequalities yield immediately that

$$
1+\left(\frac{\log 3}{\log \alpha}\right)(a-1)<n<\left(\frac{\log 3}{\log \alpha}\right) a+4
$$

If $n \leq 200$ then we have $a \leq 158$ from the above inequality. When we examine the solutions of the equation (1.4) in the Mathematica program for these bounds, we see that there is no other solution than the solutions stated in the Theorem 1. Therefore, we assume that $n>200$ from now on and from (3.4) we have that $a>158$. So, to solve the equation (1.4), it suffices to find an upper bound for $n$.

\subsection{Upper bound for $n$}

If we substitute the formula (1.2) in equation (3.1), we get $\left|\frac{\alpha^{n}}{2 \sqrt{2}}-3^{a}\right|=\left|\frac{\beta^{n}}{2 \sqrt{2}}+\frac{\alpha^{n_{1}}-\beta^{n_{1}}}{2 \sqrt{2}}-3^{a_{1}}\right|=\frac{1}{2}\left(\frac{\alpha^{n_{1}}}{\sqrt{2}}+\sqrt{2}\right)+3^{a_{1}}<3 \max \left\{\alpha^{n_{1}}, 3^{a_{1}}\right\}$. Multiplying both sides by $3^{-a}$, using the relation (3.2) and the fact that $\alpha<3$, we obtain

$$
\left|(2 \sqrt{2})^{-1} \alpha^{n} 3^{-a}-1\right|<3 \max \left\{\frac{\alpha^{n_{1}}}{3^{a}}, 3^{a_{1}-a}\right\} ; \max \left\{\alpha^{n_{1}-n+6}, 3^{a_{1}-a+1}\right\} .
$$

Let us apply the Theorem 2 to the above inequality. Set $\Delta_{1}:=(2 \sqrt{2})^{-1} \alpha^{n} 3^{-a}-1$. $\Delta_{1}$ is not zero. If it were zero, it would be $\alpha^{2 n} \in \mathbb{Z}$, which is impossible. Let us take $s:=3,\left(\gamma_{1}, \gamma_{2}, \gamma_{3}\right):=(2 \sqrt{2}, \alpha, 3)$ and $\left(b_{1}, b_{2}, b_{3}\right):=(-1, n,-a)$. We have $D:=2$ since each $\gamma_{i}$ belongs to $\mathbb{Q}(\sqrt{2})$ and we can choose $A_{1}, A_{2}, A_{3}$ and $B$ as follows:

$$
\begin{aligned}
A_{1} & :=2.1>2.079 \simeq 2 \cdot \log (2 \sqrt{2})=2 \cdot h\left(\gamma_{1}\right) \\
A_{2} & :=0.9>0.8813 \simeq \log \alpha=2 \cdot h\left(\gamma_{2}\right) \\
A_{3} & :=2.2>2.1972 \simeq 2 \cdot \log 3=2 \cdot h\left(\gamma_{3}\right) \\
B & :=2 n
\end{aligned}
$$


since $\max \{1, n, a\} \leq 2 n$. Then from Theorem 2 , we get

$$
\log \left|\Delta_{1}\right|>-1.4 \cdot 30^{6} \cdot 3^{4.5} \cdot 2^{2} \cdot(1+\log 2) \cdot(1+\log (2 n)) \cdot 2.1 \cdot 0.9 \cdot 2.2 .
$$

By comparing with (3.5), we obtain

$$
\min \left\{\left(n-n_{1}-6\right) \log \alpha,\left(a-a_{1}-1\right) \log 3\right\}<4.04 \cdot 10^{12} \cdot(1+\log (2 n))
$$

which gives

$$
\min \left\{\left(n-n_{1}\right) \log \alpha,\left(a-a_{1}\right) \log 3\right\}<4.1 \cdot 10^{12} \cdot(1+\log (2 n)) .
$$

Now, let us consider the above result as two cases.

Case 1. $\min \left\{\left(n-n_{1}\right) \log \alpha,\left(a-a_{1}\right) \log 3\right\}=\left(n-n_{1}\right) \log \alpha$.

In this case, we rewrite the equation (3.1) as

$$
\left|\left(\frac{\alpha^{n-n_{1}}-1}{2 \sqrt{2}}\right) \alpha^{n_{1}}-3^{a}\right|=\left|\frac{\beta^{n}-\beta^{n_{1}}}{2 \sqrt{2}}-3^{a_{1}}\right|<1+3^{a_{1}}<3^{1+a_{1}},
$$

which implies

$$
\left|\left(\frac{\alpha^{n-n_{1}}-1}{2 \sqrt{2}}\right) \alpha^{n_{1}} 3^{-a}-1\right|<3^{a_{1}-a+1} .
$$

Set $\Delta_{2}:=\left(\frac{\alpha^{n-n_{1}}-1}{2 \sqrt{2}}\right) \alpha^{n_{1}} 3^{-a}-1$. If $\Delta_{2}=0$, then we get

$$
\alpha^{n}-\alpha^{n_{1}}=2 \sqrt{2} \cdot 3^{a} .
$$

Conjugating this equation in $\mathbb{Q}(\sqrt{2})$ yields that

$$
\beta^{n}-\beta^{n_{1}}=-2 \sqrt{2} \cdot 3^{a}
$$

From the triangle inequality, absolute value of the left hand side of the equation (3.8) is less than 2, but that of the right hand side is greater than 2 for $a>158$. This is a contradiciton and hence $\Delta_{2} \neq 0$. So, we can apply Theorem 2 to the equation (3.7) with the parameters

$$
s=3, \quad\left(\gamma_{1}, \gamma_{2}, \gamma_{3}\right):=\left(\frac{\alpha^{n-n_{1}}-1}{2 \sqrt{2}}, \alpha, 3\right) \quad \text { and } \quad\left(b_{1}, b_{2}, b_{3}\right):=\left(1, n_{1},-a\right) .
$$

Since the minimal polynomial of $\gamma_{1}$ divides

$$
8 x^{2}-8 P_{n-n_{1}} x-\left((-1)^{n-n_{1}}+1-Q_{n-n_{1}}\right)
$$

where $\left\{Q_{t}\right\}_{t \geq 0}$ is the Pell-Lucas sequence, we get

$$
\begin{aligned}
h\left(\gamma_{1}\right) & \leq \frac{1}{2}\left(\log 8+\log \left(\frac{\alpha^{n-n_{1}}+1}{2 \sqrt{2}}\right)\right)<\frac{1}{2} \log \left(4 \sqrt{2} \alpha^{n-n_{1}}\right) \\
& <\frac{1}{2}\left(n-n_{1}+2\right) \log \alpha<4.2 \cdot 10^{11} \cdot(1+\log 2 n) .
\end{aligned}
$$

So, we can choose $A_{1}, A_{2}, A_{3}$ and $B$ as follows:

$$
A_{1}:=8.4 \cdot 10^{11} \cdot(1+\log 2 n)>2 \cdot h\left(\gamma_{1}\right)
$$




$$
\begin{aligned}
A_{2} & :=0.9>2 \cdot h\left(\gamma_{2}\right) \\
A_{3} & :=2.2>2 \cdot h\left(\gamma_{3}\right) \\
B & :=2 n
\end{aligned}
$$

since $\max \left\{1, n_{1}, a\right\} \leq 2 n$. Thus, we obtain that

$$
\log \left|\Delta_{2}\right|>-C \cdot 8.4 \cdot 10^{11} \cdot(1+\log 2 n) \cdot 0.9 \cdot 2.2
$$

where

$$
C:=1.4 \cdot 30^{6} \cdot 3^{4.5} \cdot 2^{2} \cdot(1+\log 2) \cdot(1+\log (2 n))
$$

and this inequality implies that

$$
\log \left|\Delta_{2}\right|>-1.61 \cdot 10^{24} \cdot(1+\log 2 n)^{2} .
$$

Comparing the last inequality with the ineqrality (3.7), we get that

$$
\left(a-a_{1}\right) \log 3<1.62 \cdot 10^{24} \cdot(1+\log 2 n)^{2} .
$$

Case 2. $\min \left\{\left(n-n_{1}\right) \log \alpha,\left(a-a_{1}\right) \log 3\right\}=\left(a-a_{1}\right) \log 3$.

In this case, we rewrite the equation (3.1) as

$$
\left|\frac{\alpha^{n}}{2 \sqrt{2}}-3^{a_{1}}\left(3^{a-a_{1}}-1\right)\right|=\left|\frac{\beta^{n}+\alpha^{n_{1}}-\beta^{n_{1}}}{2 \sqrt{2}}\right|<\frac{\alpha^{n_{1}}+2}{2 \sqrt{2}}<\alpha^{n_{1}}
$$

which implies

$$
\begin{aligned}
\left|\left(2 \sqrt{2}\left(3^{a-a_{1}}-1\right)\right)^{-1} \alpha^{n} 3^{-a_{1}}-1\right| & <\frac{\alpha^{n_{1}}}{3^{a}-3^{a_{1}}} \\
& <\frac{3 \alpha^{n_{1}}}{3^{a}}<3 \alpha^{n_{1}-n+4}<\alpha^{n_{1}-n+6} .
\end{aligned}
$$

from (3.2). Let

$$
\Delta_{3}:=\left(2 \sqrt{2}\left(3^{a-a_{1}}-1\right)\right)^{-1} \alpha^{n} 3^{-a_{1}}-1 .
$$

Clearly, $\Delta_{3} \neq 0$. If the opposite were true, that is, if $\Delta_{3}=0$, then $\alpha^{2 n} \in \mathbb{Z}$, which is a contradiction. In this aplication of Theorem 2, we take

$$
\begin{aligned}
s=3, & \left(\gamma_{1}, \gamma_{2}, \gamma_{3}\right) & :=\left(2 \sqrt{2}\left(3^{a-a_{1}}-1\right), \alpha, 3\right) \\
& \text { and } & \left(b_{1}, b_{2}, b_{3}\right):=\left(-1, n,-a_{1}\right) .
\end{aligned}
$$

Since the minimal polynomial of $\gamma_{1}$ is $x^{2}-8\left(3^{a-a_{1}}-1\right)^{2}$ we get,

$$
h\left(\gamma_{1}\right)=\log \left(2 \sqrt{2}\left(3^{a-a_{1}}-1\right)\right)<\left(a-a_{1}+1\right) \log 3<4.2 \cdot 10^{12} \cdot(1+\log (2 n))
$$

from (3.6). So, we can take

$$
\begin{array}{ll}
A_{1}:=8.4 \cdot 10^{12} \cdot(1+\log 2 n) & A_{3}:=2.2 \\
A_{2}:=0.9 & B:=2 n
\end{array}
$$


since $\max \left\{1, n, a_{1}\right\} \leq 2 n$. Then, we obtain

$$
\log \left|\Delta_{3}\right|>-C \cdot 8.4 \cdot 10^{12} \cdot(1+\log (2 n)) \cdot 0.9 \cdot 2.2,
$$

where

$$
C:=1.4 \cdot 30^{6} \cdot 3^{4.5} \cdot 2^{2} \cdot(1+\log 2) \cdot(1+\log (2 n))
$$

Hence,

$$
\log \left|\Delta_{3}\right|>-1.61 \cdot 10^{25} \cdot(1+\log (2 n))^{2} .
$$

Comparing the above result with the inequality (3.9), we get

$$
\left(n-n_{1}\right) \log \alpha<1.7 \cdot 10^{25} \cdot(1+\log (2 n))^{2} .
$$

So, from Case 1 and Case 2, we have

$$
\begin{aligned}
& \min \left\{\left(n-n_{1}\right) \log \alpha,\left(a-a_{1}\right) \log 3\right\}<4.1 \cdot 10^{12} \cdot(1+\log (2 n)), \\
& \max \left\{\left(n-n_{1}\right) \log \alpha,\left(a-a_{1}\right) \log 3\right\}<1.7 \cdot 10^{25} \cdot(1+\log (2 n))^{2} .
\end{aligned}
$$

Finally, we rewrite the equation (3.1) as

$$
\left|\left(\frac{\alpha^{n-n_{1}}-1}{2 \sqrt{2}}\right) \alpha^{n_{1}}-\left(3^{a}-3^{a_{1}}\right)\right|=\left|\frac{\beta^{n}-\beta^{n_{1}}}{2 \sqrt{2}}\right|<\left|\beta^{n_{1}}\right|<1 .
$$

Dividing the leftmost and rightmost sides by $\left(3^{a}-3^{a_{1}}\right)$ in the above inequality, we get

$$
\left|\left(\frac{\alpha^{n-n_{1}}-1}{2 \sqrt{2}\left(3^{a-a_{1}}-1\right)} \alpha^{n_{1}} 3^{-a_{1}}-1\right)\right|<\left|\frac{1}{\left(3^{a}-3^{a_{1}}\right)}\right| \leq \frac{3}{3^{a}} \leq 3 \alpha^{-(n-4)} \leq \alpha^{6-n}
$$

from (3.2). Now, we can apply Theorem 2 to the above inequality with

$$
\begin{array}{ll}
s=3, & \left(\gamma_{1}, \gamma_{2}, \gamma_{3}\right):=\left(\frac{\alpha^{n-n_{1}}-1}{2 \sqrt{2}\left(3^{a-a_{1}}-1\right)}, \alpha, 3\right) \\
\text { and } & \left(b_{1}, b_{2}, b_{3}\right):=\left(1, n_{1},-a_{1}\right) .
\end{array}
$$

Let us compute $h\left(\gamma_{1}\right)$ for $A_{1}$.

$$
\begin{aligned}
h\left(\gamma_{1}\right)=h\left(\frac{\alpha^{n-n_{1}}-1}{2 \sqrt{2}\left(3^{a-a_{1}}-1\right)}\right) \leq h\left(\frac{\alpha^{n-n_{1}}-1}{2 \sqrt{2}}\right)+h\left(3^{a-a_{1}}-1\right) \\
\quad<\frac{1}{2}\left(n-n_{1}+2\right) \log \alpha+\left(a-a_{1}\right) \log 3<2.1 \cdot 10^{25} \cdot(1+\log (2 n))^{2} .
\end{aligned}
$$

So, we can take

$$
\begin{array}{ll}
A_{1}:=4.2 \cdot 10^{25} \cdot(1+\log (2 n))^{2} & A_{3}:=2.2 \\
A_{2}:=0.9 & B:=2 n
\end{array}
$$

since $\max \left\{1, n_{1}, a_{1}\right\} \leq 2 n$. If we take

$$
\Delta_{4}:=\frac{\alpha^{n-n_{1}}-1}{2 \sqrt{2}\left(3^{a-a_{1}}-1\right)} \alpha^{n_{1}} 3^{-a_{1}}-1
$$


one can easily see that $\Delta_{4} \neq 0$ as in the previous ones. So, from the Theorem 2, we obtain

$$
\log \left|\Delta_{4}\right|>-C \cdot 4.2 \cdot 10^{25} \cdot(1+\log (2 n))^{2} \cdot 0.9 \cdot 2.2
$$

where $C:=1.4 \cdot 30^{6} \cdot 3^{4.5} \cdot 2^{2} \cdot(1+\log 2) \cdot(1+\log (2 n))$. This bound and (3.11) gives

$$
n-6<8.06 \cdot 10^{37} \cdot 4.2 \cdot 10^{25} \cdot(1+\log (2 n))^{3}
$$

which can be simplified as

$$
n<8.52 \cdot 10^{43} \text {. }
$$

Now, let us try to reduce the bound on $n$.

\subsection{Reducing the bound on $n$}

We want to improve the bound on $n$ we find in (3.12) until it is small enough. We will use Lemma 1 several times for this. Let us go back to the inequality (3.5). Set

$$
\Gamma:=n \log \alpha-a \log 3-\log (2 \sqrt{2}) .
$$

Assume that $\min \left\{n-n_{1}, a-a_{1}\right\} \geq 20$. If we consider $\Delta_{1}, \Delta_{2}$ and $\Delta_{3}$ again then since we assume that $\min \left\{n-n_{1}, a-a_{1}\right\} \geq 20$ we obtain

$$
\left|e^{\Gamma}-1\right|=\left|\Delta_{1}\right|<\frac{1}{4}
$$

which implies

$$
|\Gamma|<\frac{1}{2}
$$

Since the inequality $|\xi|<2\left|e^{\xi}-1\right|$ holds for all $\xi \in\left(-\frac{1}{2}, \frac{1}{2}\right)$, we get

$$
|\Gamma|<2 \max \left\{\alpha^{n_{1}-n+6}, 3^{a_{1}-a+1}\right\} \leq \max \left\{\alpha^{n_{1}-n+7}, 3^{a_{1}-a+2}\right\} .
$$

Let us assume that $\Gamma>0$. Then we have

$$
\begin{aligned}
0 & <n\left(\frac{\log \alpha}{\log 3}\right)-a-\frac{\log (2 \sqrt{2})}{\log 3}<\max \left\{\alpha^{n_{1}-n+7}, 3^{a_{1}-a+2}\right\} \\
& <\max \left\{\frac{\alpha^{7}}{(\log 3) \cdot \alpha^{n-n_{1}}}, \frac{9}{(\log 3) \cdot 3^{a-a_{1}}}\right\}<\max \left\{436 \cdot \alpha^{-\left(n-n_{1}\right)}, 9 \cdot 3^{-\left(a-a_{1}\right)}\right\} .
\end{aligned}
$$

Now, we apply Lemma 1 with

$$
\gamma:=\frac{\log \alpha}{\log 3}, \quad \mu:=\frac{\log (1 /(2 \sqrt{2}))}{\log 3}, \quad(A, B):=(436, \alpha) \quad \text { or } \quad(A, B):=(9,3) .
$$

We can choose $M:=8.52 \cdot 10^{43}>n$ from the inequality (3.12). Computing 86th convergent of $\gamma$ with the help of the Mathematica gives

$$
q:=525422721605994540683823357818903486740995467
$$


where $q>6 M$. For this value of $q$, it can be seen that $\varepsilon>0.35$. So, we have either

$$
n-n_{1}<125 \quad \text { or } \quad a-a_{1}<97 \text {. }
$$

In case of $\Gamma<0$, variables change as

$$
\gamma:=\frac{\log 3}{\log \alpha}, \quad \mu:=\frac{\log (2 \sqrt{2})}{\log \alpha}, \quad(A, B):=(543, \alpha) \quad \text { or } \quad(A, B):=(11,3) .
$$

So, we get $\varepsilon>0.42$ but this does not affect the result stated in (3.13).

Let us consider separately the results obtained in the inequality (3.13).

Firstly, let $n-n_{1}<125$. Assumed that $a-a_{1} \geq 20$ and consider the inequality (3.7). Set

Then, we get

$$
\Gamma_{1}:=n_{1} \log \alpha-a \log 3+\log \left(\frac{\alpha^{n-n_{1}}-1}{2 \sqrt{2}}\right) .
$$

$$
\left|\Gamma_{1}\right|<\frac{6}{3^{a-a_{1}}}
$$

from the inequality (3.7). Let us assume that $\Gamma_{1}>0$. In this case, we have

$$
0<n_{1}\left(\frac{\log \alpha}{\log 3}\right)-a+\frac{\log \left(\left(\alpha^{n-n_{1}}-1\right) /(2 \sqrt{2})\right)}{\log 3}<\frac{6}{(\log 3) \cdot 3^{a-a_{1}}}<\frac{6}{3^{a-a_{1}}}
$$

Let we again apply the Lemma 1 with the parameters

$$
\gamma:=\frac{\log \alpha}{\log 3}, \quad \mu:=\frac{\log \left(\left(\alpha^{n-n_{1}}-1\right) /(2 \sqrt{2})\right)}{\log 3}, \quad(A, B):=(6,3)
$$

for $n-n_{1} \in\{1,2, \cdots 124\}$. All values of $\varepsilon$ are greater than 0.00046 for the 88th convergent of $\gamma$ which also satisfies the condition $q>6 M$. So, we get

$$
a-a_{1}<\frac{\log (6 q / 0.00046)}{\log 3}<106 .
$$

Thus, $n-n_{1}<125$ implies that $a-a_{1}<106$. In case $\Gamma_{1}<0$, the same results are obtained.

Secondly, now, let us assumed that $a-a_{1}<97$. Set

$$
\Gamma_{2}:=n \log \alpha-a_{1} \log 3+\log \left(1 /\left(2 \sqrt{2}\left(3^{a-a_{1}}-1\right)\right)\right)
$$

from the inequality (3.9) for $\Delta_{3}$ and assume that $n-n_{1} \geq 20$. Hence, we get

$$
\left|\Gamma_{2}\right|<\frac{3 \alpha^{6}}{\alpha^{n-n_{1}}} \text {. }
$$

Assume that $\Gamma_{2}>0$. Then, we obtain

$$
0<n\left(\frac{\log \alpha}{\log 3}\right)-a_{1}+\frac{\log \left(1 /\left(2 \sqrt{2}\left(3^{a-a_{1}}-1\right)\right)\right)}{\log 3}<\frac{3 \alpha^{6}}{\log 3 \cdot \alpha^{n-n_{1}}}<\frac{541}{\alpha^{n-n_{1}}} \text {. }
$$


If we again apply Lemma 1 with the parameters

$$
\gamma:=\frac{\log \alpha}{\log 3}, \quad \mu:=\frac{\log \left(1 /\left(2 \sqrt{2}\left(3^{a-a_{1}}-1\right)\right)\right)}{\log 3}, \quad(A, B):=(541, \alpha)
$$

for $a-a_{1} \in\{1,2, \cdots, 96\}$ we get $\varepsilon>0.005$ and

$$
n-n_{1}<134 .
$$

The same result is obtained for $\Gamma_{2}<0$. Hence, if $n-n_{1}<125$, then $a-a_{1}<106$, and if $a-a_{1}<97$, then $n-n_{1}<134$. Consequently, the results $n-n_{1} \leq 134$ and $a-a_{1} \leq 105$ are always valid.

Finally, set

$$
\Gamma_{3}:=n_{1} \log \alpha-a_{1} \log 3+\log \left(\frac{\alpha^{n-n_{1}}-1}{2 \sqrt{2}\left(3^{a-a_{1}}-1\right)}\right)
$$

using the inequality (3.11). Since $n>200$, inequality (3.11) implies that

$$
\left|\Gamma_{3}\right|<\frac{3}{\alpha^{n-6}}=\frac{3 \alpha^{6}}{\alpha^{n}} .
$$

Assume that $\Gamma_{3}>0$. Then, we have

$$
0<n_{1}\left(\frac{\log \alpha}{\log 3}\right)-a_{1}+\frac{\log \left(\left(\alpha^{n-n_{1}}-1\right) /\left(2 \sqrt{2}\left(3^{a-a_{1}}-1\right)\right)\right)}{\log 3}<\frac{3 \alpha^{6}}{\log 3 \cdot \alpha^{n}}<\frac{541}{\alpha^{n}} .
$$

If we again apply Lemma 1 to the above inequality for $n-n_{1} \in\{1, \cdots, 134\}$ and $a-a_{1} \in\{1, \cdots, 105\}$ except for $\left(n-n_{1}, a-a_{1}\right)=(4,1)$ we get $\varepsilon>6.70754 \times 10^{-6}$ and

$$
n<146 .
$$

A similar result is obtained for $\Gamma_{3}<0$. Now, let $\left(n-n_{1}, a-a_{1}\right)=(4,1)$. Since

$$
\frac{\alpha^{4}-1}{4 \sqrt{2}}=\alpha^{2}
$$

we have

$$
\Gamma_{3}:=\left(n_{1}+2\right) \log \alpha-a_{1} \log 3 .
$$

Hence, we get

$$
\left|\gamma-\frac{a_{1}}{n_{1}+2}\right|<\frac{541}{\left(n_{1}+2\right) \alpha^{n}} .
$$

Since $n>200, \alpha^{n}>2 \times 541 \times\left(9 \times 10^{43}\right)>2 \times 541 \times\left(n_{1}+2\right)$ which implies that right hand side of above inequality is at most $2 /\left(n_{1}+2\right)^{2}$. Let $\left[s_{0}, s_{1}, s_{2}, \cdots\right]=$ $[0,1,4, \cdots]$ be the continued fraction expression of the above $\gamma$ and let $p_{t} / q_{t}$ be its $t$ th convergent. By Legendre's criterion, we obtain that $a_{1} /\left(n_{1}+2\right)=p_{t} / q_{t}$ for 
some $t \in\{1,2, \cdots, 85\}$. Here we use that $n_{1}+2<9 \times 10^{43}<q_{85}$. Then putting $s(M):=\max \left\{s_{i}: i=1, \cdots, 85\right\}=181$ we get

$$
\left|\gamma-\frac{p_{t}}{q_{t}}\right|>\frac{1}{(s(M)+2) q_{t}^{2}}=\frac{1}{183 q_{t}^{2}} .
$$

Therefore, we obtain

which implies that

$$
\frac{1}{183 q_{t}^{2}}<\frac{541}{\alpha^{n} q_{t}}
$$

$$
\alpha^{n}<541 \times 183 \times 9 \times 10^{43} .
$$

So, we get

$$
n<128 .
$$

Bounds for $n$ in (3.14) and (3.15) are all contradicts our assumption that $n>200$. This completes the proof of Theorem 1 .

\section{ACKNOWLEDGEMENT}

The author expresses his gratitude to the reviewers for their contribution to improving the readability of the article.

Conflicts of interest/Competing interests: The authors declare that they have no conflict of interest.

\section{REFERENCES}

[1] A. Baker and H. Davenport, "The equations $3 x^{2}-2=y^{2}$ and $8 x^{2}-7=z^{2}$," The Quarterly Journal of Mathematics, vol. 20, no. 1, pp. 129-137, 1969, doi: 10.1093/qmath/20.1.129.

[2] A. Baker and G. Wüstholz, Logarithmic forms and Diophantine geometry. Cambridge University Press, 2008. doi: 10.1017/CBO9780511542862.

[3] J. J. Bravo, F. Luca, and K. Yazan, "On Pillai's problem with Tribonacci numbers and Powers of 2," Bull. Korean Math. Soc, vol. 54, no. 3, pp. 1069-1080, 2017, doi: 10.4134/BKMS.b160486.

[4] J. J. Bravo and F. Luca, "On a conjecture about repdigits in k-generalized Fibonacci sequences," Publ. Math. Debrecen, vol. 82, no. 3-4, pp. 623-639, 2013, doi: 10.5486/PMD.2013.5390.

[5] Y. Bugeaud, M. Mignotte, and S. Siksek, "Classical and modular approaches to exponential Diophantine equations I. Fibonacci and Lucas perfect powers," Annals of Mathematics, pp. 969-1018, 2006, doi: 10.4007/annals.2006.163.969.

[6] K. C. Chim, I. Pink, and V. Ziegler, "On a variant of Pillai's problem," International Journal of Number Theory, vol. 13, no. 4, pp. 1711-1727, 2017, doi: 10.1142/S1793042117500981.

[7] K. C. Chim, I. Pink, and V. Ziegler, "On a variant of Pillai's problem II," Journal of number theory, vol. 183, pp. 269-290, 2018, doi: 10.1016/j.jnt.2017.07.016.

[8] M. Ddamulira, "On a problem of Pillai with Fibonacci numbers and powers of 3," Bol. Soc. Mat. Mex., vol. 26, no. 2, pp. 263-277, 2019, doi: 10.1007/s40590-019-00263-1.

[9] M. Ddamulira, "On the problem of Pillai with Tribonacci numbers and powers of 3," Journal of Integer Sequences, vol. 22, no. 5, 2019.

[10] M. Ddamulira, C. A. Gómez, and F. Luca, "On a problem of Pillai with k-generalized Fibonacci numbers and powers of 2," Monatshefte für Mathematik, vol. 187, no. 4, pp. 635-664, 2018, doi: 10.1007/s00605-018-1155-1. 
[11] M. Ddamulira and F. Luca, "On the problem of Pillai with k-generalized Fibonacci numbers and powers of 3," International Journal of Number Theory, vol. 16, no. 7, pp. 1643-1666, 2020, doi: $10.1142 / \mathrm{S} 1793042120500876$.

[12] M. Ddamulira, "On the problem of Pillai with Padovan numbers and powers of 3," Studia Scientiarum Mathematicarum Hungarica, vol. 56, no. 3, pp. 364-379, 2019, doi: 10.1556/012.2019.56.3.1435.

[13] M. Ddamulira, F. Luca, and M. Rakotomalala, "On a problem of Pillai with Fibonacci numbers and powers of 2," Proceedings-Mathematical Sciences, vol. 127, no. 3, pp. 411-421, 2017, doi: 10.1007/s12044-017-0338-3.

[14] A. Dujella and A. Petho, "A generalization of a theorem of Baker and Davenport," The Quarterly Journal of Mathematics, vol. 49, no. 195, pp. 291-306, 1998, doi: 10.1093/qmathj/49.3.291.

[15] A. C. García Lomelí and S. Hernández Hernández, "Pillai’s problem with Padovan numbers and powers of two," Revista Colombiana de Matemáticas, vol. 53, no. 1, pp. 1-14, 2019, doi: 10.15446/recolma.v53n1.81034.

[16] A. C. García Lomelí, S. Hernández Hernández, and F. Luca, "Pillai’s problem with the Fibonacci and Padovan sequences," in Annales Mathematicae et Informaticae, vol. 50, doi: 10.33039/ami.2019.09.001. Eszterházy Károly Egyetem Líceum Kiadó, 2019, pp. 101-115.

[17] A. C. García Lomelí, S. Hernández Hernández, and F. Luca, "Pillai's problem with the Padovan and Tribonacci sequences," Indian Journal of Mathematics, vol. 61, no. 1, pp. 61-75, 2019.

[18] S. Hernández Hernández, F. Luca, and L. M. Rivera, "On Pillai’s problem with the Fibonacci and Pell sequences," Boletín de la Sociedad Matemática Mexicana, vol. 25, no. 3, pp. 495-507, 2019, doi: 10.1007/s40590-018-0223-9.

[19] M. O. Hernane, F. Luca, S. Rihane, and A. Torbé, "On Pillai's problem with Pell numbers and powers of 2," Hardy Ramanujan Journal, vol. 41, pp. 22-31, 2019, doi: 10.46298/hrj.2019.5102.

[20] A. Herschfeld, "The equation $2^{x}-3^{y}=d$," Bulletin of the American Mathematical Society, vol. 41, no. 9, p. $631,1935$.

[21] A. Herschfeld, "The equation $2^{x}-3^{y}=d$," Bulletin of the American Mathematical Society, vol. 42, no. 4, pp. 231-234, 1936.

[22] M. Liana, A. Szynal-Liana, and I. Wloch, "On Pell hybrinomials," Miskolc Mathematical Notes, vol. 20, no. 2, pp. 1051-1062, 2019, doi: 10.18514/MMN.2019.2971.

[23] E. M. Matveev, "An explicit lower bound for a homogeneous rational linear form in the logarithms of algebraic numbers. ii," Izvestiya: Mathematics, vol. 64, no. 6, p. 1217, 2000, doi: 10.1070/IM2000v064n06ABEH000314.

[24] P. Mihailescu, "Primary cyclotomic units and a proof of Catalans conjecture," Journal für die reine und angewandte Mathematik, vol. 572, pp. 167-195, 2006, doi: 10.1515/crll.2004.048.

[25] S. Pillai, "On $a^{x}-b^{y}=c$," J. Indian Math. Soc, vol. 2, pp. 119-122, 1936.

[26] S. Pillai, "A correction to the paper on $a^{x}-b^{y}=c$," J. Indian Math. Soc.(NS), vol. 2, p. 215, 1937.

[27] N. P. Smart, The algorithmic resolution of Diophantine equations: a computational cookbook. Cambridge University Press, 1998, vol. 41, doi: 10.1017/CBO9781107359994.

[28] R. Stroeker and R. Tijdeman, "Diophantine equations, in Computational Methods in Number Theory, Part II," Mathematics Centre Tracts, vol. 155, pp. 231-234, 1935.

Author's address

A. Çă̆man

Ağrı İbrahim Çeçen University, Department of Mathematics, 12 Curly St., 04100 Ağrı, Turkey

E-mail address: acagman@agri.edu.tr 